\title{
稳定同位素标记试剂DiART在蛋白质组中的定量 特征研究
}

\author{
姜翠翠 ${ }^{1}$, 吕晓磊 ${ }^{1}$, 吴迪 ${ }^{1}$, 李杨 $^{1}$, 董宇升 ${ }^{1}, 弓^{2}$ 纪彬 ${ }^{1}$, 王全会 ${ }^{1,2^{*}}$ \\ 1. 北京华大蛋白质研发中心有限公司, 北京 101318 ; \\ 2. 中国科学院大学中丹学院, 北京 100049 \\ *联系人, E-mail: wangqh@genomics.cn \\ 收稿日期: 2017-10-16; 接受日期: 2017-12-27; 网络版发表日期: 2018-02-13 \\ 国家重点基础研究发展计划(批准号: 2010CB912703)和北京市自然科学基金(批准号: 5132023)资助
}

摘要 等重同位素标记方法, 如同位素标记相对和绝对定量(iTRAQ), 可以对肽段进行化学标记, 之后通过质谱分 析得到的报告离子的强度信息而实现对肽段的定量. 目前，这种标记技术在定量蛋白质组学研究中有广泛应用. DiART也是一种等重同位素标记方法, 且与iTRAQ的定量原理类似, 但是其化学结构组成与iTRAQ有所不同, 因 而其定量特征有其独特表现. 本研究通过对DiART标记的简单蛋白样品、复杂蛋白样品以及复杂样品中目标蛋 白的定量情况进行了分析, 从而对DiART的定量特征进行了研究, 并同时与iTRAQ进行了比较. 结果表明, DiART方法在定量稳定性、准确性及动态范围方面均更具优势.

关键词 DiART, iTRAQ, 定量蛋白质组

定量蛋白质组技术因其能够对蛋白质的表达丰度 进行规模化分析, 一直是蛋白质组学在生命科学领域 的重要应用方向. 总体而言, 定量蛋白质组技术包括 非标记定量技术(label-free)和标记定量技术. 前者是 不对蛋白或肽段进行标记，而基于肽段质谱图数量、 色谱峰面积等参数获取定量信息的方法，后者则是通 过对蛋白或肽段进行稳定同位素标记，之后根据标记 肽段或同位素标签的质谱信号获取定量信息的方 法 ${ }^{[1,2]}$. 相比于非标记定量技术而言，基于稳定同位素 标记的技术可以将标记后的样本进行混合, 通过一次 质谱分析即可实现对多重样本的同时比较，因而可以 在很大程度上降低实验误差，实现更为精准的定量分
析和获取更为可信的定量结果. 因此，基于稳定同位 素标记技术的定量蛋白质组研究在近年来得到了快速 的发展 ${ }^{[3,4]}$. 细胞培养条件下稳定同位素标记技术 (stable isotope labeling with amino acids in cell culture, SILAC) ${ }^{[5]}$ 、同位素标记相对和绝对定量(isobaric tags for relative and absolute quantification, iTRAQ $)^{[6]}$ 和同 量异序标签 (tandem mass tag, TMT) ${ }^{[7]}$ 为其中最具代表 性的稳定同位素标记技术, 此外还有 $N, N$-二甲基亮氨

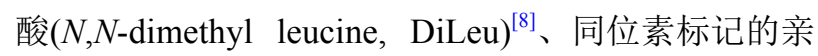
和标签(isotope-coded affinity tag, ICAT) ${ }^{[9]}$ 、同位素标 记蛋白技术(isotope coded protein labeling, ICPL) ${ }^{[10]}$ 、 ${ }^{18} \mathrm{O}^{[11]}$ 等.

引用格式: 姜翠翠, 吕晓否, 吴迪, 等. 稳定同位素标记试剂DiART在蛋白质组中的定量特征研究. 中国科学: 生命科学, 2018, 48: 224-231 Jiang C C, Lv X L, Wu D, et al. Evaluation of the use of DiART isobaric labeling tags in quantitative proteomics studies (in Chinese). Sci Sin Vitae, 2018, 48: 224-231, doi: 10.1360/N052017-00152 
以SILAC，iTRAQ和TMT为代表的标记技术各有 其特点. 其中SILAC是一种代谢标记技术，通过在培 养基中掺入重标的必需氨基酸, 从而在细胞培养过程 中实现对蛋白质的标记. 最常用的标记氨基酸为精氨 酸和赖氨酸, 还有甲硫氨酸、亮氨酸和酪氨酸, 通过 这些不同标记氨基酸的组合，可以实现多重定量比较. SILAC技术的优点是样本可在细胞水平进行混合，减 少了在蛋白提取、酶解和质谱检测多个阶段的实验误 差, 应能得到更为准确的定量结果. 然而, 该类方法仅 适用于活体细胞标记, 对于各种临床病理样本、植物 样本等则不适用. 近年来, 有些研究者通过用含重标 氨基酸的特殊饲料饲喂实验动物, 进行活体动物的SI$\mathrm{LAC}$ 标记, 使这种技术拓展到动物模型研究中, 将这一 技术的应用范围大大拓宽，然而其代价的昂贵也是显 而易见的 ${ }^{[12 \sim 14]}$. iTRAQ和TMT则是对蛋白或肽段的伯 氨基进行体外化学标记，不受限于活体培养、不局限 于样本类型，因而具有更好的普适性，应用领域较SILAC更为广泛。该类技术目前可以最多实现对 10 组样 品的同时比较. 另外, iTRAQ和TMT技术的特点是等 重标记，仅在二级质谱水平体现多重同位素标签的差 异，也即其定量信息是在二级质谱水平提取的，排除 了一级质谱信号的复杂干扰，提高了定量的准确度. 正因如此, 目前以iTRAQ和TMT为标记方法的定量蛋 白质组研究获得了更多的应用.

DiART (deuterium isobaric amine reactive tag) 是一 种可对蛋白质/肽段的伯氨基进行稳定同位素标记的 方法，其标记和定量原理与iTRAQ相似，同为等重标 记，并通过二级质谱同位素报告离子峰的强度信息进 行定量比较 ${ }^{[15]}$. DiART同样包含氨基反应基团、平衡 基团和报告基团. 报告基团的 $\mathrm{m} / \mathrm{z}$ 为 $114,115,116,117$, 118，119, 共6个通道. 与iTRAQ的不同之处在于, iTRAQ报告基团是基于 $N, N^{\prime}$-烷基化甘氨酸，而DiART 是基于 $N, N^{\prime}$-二甲基亮氨酸 ${ }^{[16,17]}$. 更为重要的是, DiART 试剂中稳定同位素采用了 ${ }^{2} \mathrm{H}$, 减少了 ${ }^{13} \mathrm{C}$ 和 ${ }^{15} \mathrm{~N}$ 的数目, 从而大大降低了合成成本并且比较容易合成 ${ }^{[15]}$.

虽然引入 ${ }^{2} \mathrm{H}$ 会带来一定的色谱偏移而影响定量的 准确性, 但是研究发现, 通过使用最小数量的 ${ }^{2} \mathrm{H}$ 原子, 并将其设置在亲水基团如叔胺附近，可以降低 ${ }^{2} \mathrm{H}$ 原子 的同位素效应，降低在不同通道中 ${ }^{2} \mathrm{H}$ 数量的不同可能 带来的标记肽段亲水性差异. 实验结果表明, DiART 因引入 ${ }^{2} \mathrm{H}$ 元素造成的肽段在质谱和色谱水平的差异可
以忽略不计 ${ }^{[18]}$. 进一步研究表明, 在同样的质谱参数条 件下，DiART标记后的肽段所产生的二级质谱峰报告 离子的相对强度高于iTRAQ所产生的报告离子 ${ }^{[19]}$. 在 不影响肽段鉴定的情况下，报告离子相对强度较高， 可以提高定量结果的准确度，同时可以提高定量的灵 敏度. 之前的研究显示, 相对于iTRAQ, DiART标记方 法对于同样的样品可以得到更多的差异蛋白，而对于 同一个蛋白用DiART方法所得到的差异倍数明显高于 用iTRAQ方法所得到的差异倍数 ${ }^{[20]}$.

此前虽然对DiART用于蛋白质定量分析进行了一 些研究, 但是缺少对定量结果准确性的验证. 本研究从 已知定量比值的样品着手, 对DiART标记的简单样 品、复杂样品, 以及复杂样品中的目标蛋白进行了分 析, 以进一步评价DiART的定量特征. 研究发现, 相比 于iTRAQ方法, DiART标记方法的定量稳定性和准确 性均具有一定的优势, 并且复杂背景的干扰对其影响 较小, 可以在一定程度上克服iTRAQ存在的定量比值 低估问题.

\section{1 材料与方法}

\section{1 试剂及材料}

DiART试剂(合作实验室化学合成)、iTRAQ试剂 (AB-SCIEX, 美国)、BSA肽段(Bruker, 德国)、大肠杆 菌蛋白、Micro-QTOF质谱仪(Bruker)、纳升级高效液 相色谱(Thermo-scientific, 美国).

\section{2 蛋白提取及酶解}

将大肠杆菌菌体用PBS缓冲液清洗3遍，之后用一 定体积裂解液 $(8 \mathrm{~mol} / \mathrm{L}$ 尿素, $30 \mathrm{mmol} / \mathrm{L}$ HEPES, $1 \mathrm{mmol} / \mathrm{L}$ PMSF, $2 \mathrm{mmol} / \mathrm{L}$ EDTA, $10 \mathrm{mmol} / \mathrm{L}$ DTT, $50 \mathrm{mmol} / \mathrm{L}$ Tris- $\mathrm{Cl}$ )重悬菌体并进行超声辅助裂解. 将 得到的细菌裂解液进行离心 $\left(6000 \times g, 5 \mathrm{~min}, 4^{\circ} \mathrm{C}\right)$ 去除 残留菌体, 上清即为大肠杆菌全蛋白. 将大肠杆菌全蛋 白进行蛋白定量, 之后取一定量的蛋白溶液进行Tryp$\mathrm{sin}$ 酶解。酶解过程是首先加入 D T T 至终浓度 $10 \mathrm{mmol} / \mathrm{L}$, 于 $56^{\circ} \mathrm{C}$ 水浴 $1 \mathrm{~h}$, 然后在 $55 \mathrm{mmol} / \mathrm{L} \mathrm{IAM}$ 条 件下，置于暗室 $1 \mathrm{~h}$ 进行烷基化反应。还原烷基化后的 蛋白溶液用 $25 \mathrm{mmol} / \mathrm{L} \quad \mathrm{NH}_{4} \mathrm{HCO}_{3}$ 溶液稀释4倍, 按照 30:1 (蛋白:酶)的比例加入 Trypsin酶, 于 $37^{\circ} \mathrm{C}$ 反应 $24 \mathrm{~h}$. 之后再按照 100:1的比例补加Trypsin酶, 继续反应 $12 \mathrm{~h}$. 


\section{3 肽段标记}

将获得的大肠杆菌全蛋白酶解肽段溶液分成两部 分, 分别进行真空抽干. 将获得的肽段干粉首先分别用 一定体积的 $1 \mathrm{~mol} / \mathrm{L} \mathrm{TEAB}$ 重新溶解, 然后在肽段溶液 中分别加入一定体积异丙醇并混匀，使异丙醇的体积 比不低于 $60 \%$. 最后在肽段溶液中分别加入DiART117, DiART-118和iTRAQ-117, iTRAQ-118试剂, 室温 反应 $1 \mathrm{~h}$. 将标记后的肽段按照不同比例混合，其中, BSA-DiART-117和BSA-DiART-118肽段按照1:1，1:2, 1:3, 1:4, 1:5, 1:8, 1:10混合, BSA-iTRAQ-117和BSAiTRAQ-118肽段同样按照1:1１:2，1:3，1:4，1:5，1:8, $1: 10$ 混合. 混合后的肽段样品用Zip-Tip柱进行除盐.

\subsection{LC-MS/MS分析}

取一定量制备好的肽段进行质谱分析. 在液相系 统中, 肽段样品先以流速为 $4 \mu \mathrm{L} / \mathrm{min}$ 的流速载入预柱 (Acclaim PePmap 100, $75 \mu \mathrm{m} \times 2 \mathrm{~cm}$, nanoviper, $\mathrm{C}^{18}$, $3 \mu \mathrm{m}, 100 \mathrm{~A}$, Thermo scientific), 再以 $400 \mathrm{~nL} / \mathrm{min}$ 的流速 在自制纳升喷针一体柱(Venusil $\times \mathrm{BPC}, \mathrm{C}^{18}, 5 \mu \mathrm{m}$, 150A, Agela Technologies, 天津)上进行分离. 液相梯 度设置为: $5 \%$ 乙腈, $5 \mathrm{~min} ; 5 \%$ 28\%乙腈, $30 \mathrm{~min} ; 28 \%$ 40\%乙腈，10 min; 40\% 80\%乙腈，5 min； 80\%乙腈， $5 \mathrm{~min} ; 80 \% \sim 5 \%$ 乙腈, $5 \mathrm{~min}$.

质谱仪离子源毛细管电压为 $1500 \mathrm{~V}$, 干燥气流速 为 $2.5 \mu \mathrm{L} / \mathrm{min}$, 温度为 $150^{\circ} \mathrm{C}$. 质谱数据采集方式为数 据依赖型采集(Data-dependent MS/MS acquisition). 质 谱扫描范围为 $50 \sim 22000 \mathrm{Da}$ ，扫描速度 $4.0 \mathrm{~Hz}$, 碰撞池 碰撞能量为 $8 \mathrm{eV}$, RF电压设置为 $500 \sim 150 \mathrm{vpp}$, 传输时 间为 $90 \mu \mathrm{s}$.

每个样品进行 3 次质谱分析, 鉴定肽段数及定量值 取平均值.

\section{5 数据及定量分析}

数据搜索和定量软件采用Mascot (2.3.1). 数据库 为BSA自建库和Swissport-E. coli (2015年6月更新). 一 级质谱和二级质谱的容错范围均为 $0.05 \mathrm{Da}$, 固定修饰 为Carbamidomethyl $(\mathrm{C})$ : 可变修饰为: Oxidation $(\mathrm{M})$, iTRAQ (K), iTRAQ (N-term), DiART(K), DiART (Nterm), Deamidation (N, Q). 定量选择iTRAQ-8 plex或者 DiART.

\section{2 结果}

\subsection{Micro-QTOF质谱检测DiART和iTRAQ标记 肽段的参数优化}

iTRAQ和DiART标记肽段在质谱检测时产生的子 离子主要包括两个部分, 肽段本身的碎片和标记试剂 的报告离子. 在二级谱峰中报告离子的强度比例太高 会影响肽段的鉴定，太低则会影响肽段的定量效果， 因此应该将其控制在一个相对合理的范围内. 由于报 告离子 $\mathrm{m} / \mathrm{z}$ 为 $113 \sim 121$, 处于低质量端, 因此可以通过 对低 $\mathrm{m} / \mathrm{z}$ 离子传输效率的调整来控制其强度比例. 在 QTOF质谱仪中, 在肽段得到充分碎裂的条件下, 四级 杆离子射频电压(radio frequency, RF) 是控制不同 $\mathrm{m} / \mathrm{z}$ 离子传输效率进而影响离子强度的重要参数. 因此, 本研究对micro-QTOF碰撞池四级杆RF电压进行了不 同的设置，以检测最适合iTRAQ和DiART标记肽段鉴 定和定量的质谱条件.

将RF电压设置在150 500 vpp动态变化, 将 $90 \mu \mathrm{s}$ 的离子传输时间与 RF值的动态变化进行关联, 设定不 同的分配比例. 低质量端离子传输时间分配分别设置 了 $15 \%, 20 \%, 25 \%, 30 \%, 35 \%, 40 \%, 45 \%, 50 \%$, 相对 应的高质量端离子传输时间分别设置了 $85 \%, 80 \%$, $75 \%, 70 \%, 65 \%, 60 \%, 55 \%, 50 \%$. 如此, 共有8个质谱 条件, 分别命名为方法1 方法8. 将用iTRAQ和DiART 标记的BSA肽段样品分别在 8 个质谱条件下进行了检 测. 经过数据库搜索后, 分别对鉴定的肽段数目及含有 报告离子的肽段数目进行了统计. 结果如图1所示. 对 于DiART，在方法 3 条件下鉴定肽段数目最多，为 151 条, 方法6和方法7次之, 为 149 条; 在方法 6 条件下可定 量肽段数目最多(即含报告离子的肽段), 为 145 条, 方 法7次之, 为 143 条, 因此综合而言, 方法6比较适合 DiART标记肽段的检测. 对于iTRAQ, 在方法3条件下 肽段鉴定数目最多, 为 145 条; 在方法 2 , 方法 3 条件下可 定量肽段数目最多, 为 138 条, 因此综合而言, 方法 3 比 较适合iTRAQ标记肽段的检测.

\subsection{DiART标记方法的定量准确度和动态范围 分析}

采用上述最优质谱条件，分别对DiART和iTRAQ 标记的BSA肽段各7个样品进行分析. 如方法部分所 述, DiART标记的 7 个样品分别由DiART117和 

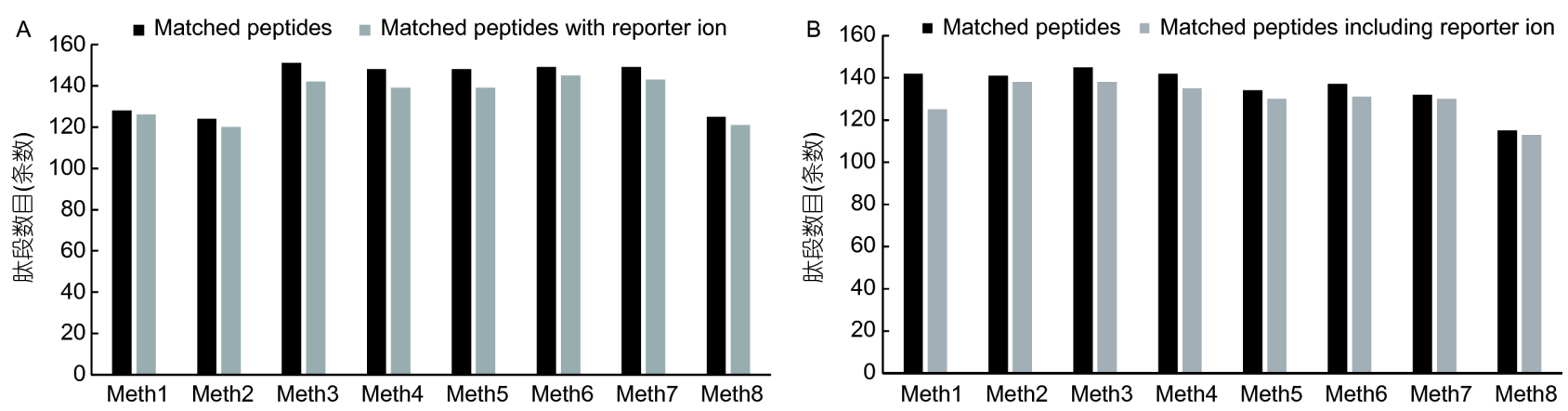

图 1 不同质谱方法对应的肽段鉴定和定量数目

A: DiART标记肽段的结果; B: iTRAQ标记肽段的鉴定结果

DiART118标记肽段以 $1: 1,1: 2,1: 3,1: 4,1: 5,1: 8,1: 10$ 混 合得到; iTRAQ标记的7个样品分别由iTRAQ117和 iTRAQ118标记肽段以 $1: 1,1: 2,1: 3,1: 4,1: 5,1: 8,1: 10$ 混合得到. 经过Mascot鉴定和定量分析, 将每个肽段的 定量比值与理论值相比较, 分析DiART标记定量方法 的准确性. 可以看出, 无论DiART还是iTRAQ标记的 肽段, 对于理论比值为 $1: 5$ 以内的肽段, 其定量结果与 理论值均比较接近, 而对于理论比值为 $1: 8$ 和 $1: 10$ 这样 的肽段，DiART方法得到的定量比值比iTRAQ方法更 接近理论值, 说明DiART在降低比值压缩方面更有优 势(图2). 另一方面, DiART标记肽段定量结果的变异 度明显低于iTRAQ, 针对理论比值 $1: 1,1: 2,1: 3,1: 4$, 1:5, 1:8, 1:10的样品, DiART标记肽段的变异系数分别 为 $26.38 \%, 37.99 \%, 24.94 \%, 31.55 \%, 26.88 \%, 33.23 \%$, $35.72 \%$, 而iTRAQ标记样品的变异系数分别为 $42.43 \%$, $44.44 \%, 42.18 \%, 37.89 \%, 49.89 \%, 44.55 \%, 42.69 \%$, 说 明DiART标记定量方法更为稳定.

\subsection{DiART对复杂样品肽段的定量稳定性分析}

为了进一步检查DiART对复杂样品的定量分析能 力, 用DiART标记了大肠杆菌肽段, 同时以iTRAQ标记 的样品作为对照. 大肠杆菌肽段分别以DiART114和 DiART115标记, 之后以 1:4混合, iTRAQ标记的样品采 取相同处理. 样品分别经质谱检测和Mascot搜库鉴定 及定量分析. 根据搜库结果, DiART和iTRAQ标记的 样品分别定量到 1292 和 2428 个大肠杆菌肽段, 以及 484 和 571 个大肠杆菌蛋白. 之后在.mgf文件中提取所 有报告离子的强度值, 并进行统计. 结果表明, DiART 报告离子的强度主要分布在 $10^{5}$, 在 $10^{6}$ 及以上数量级 上也有较高比例, 而iTRAQ报告离子强度则主要在 $10^{5}$
及以下, 说明对于同样的肽段, DiART报告离子强度普 遍高于iTRAQ, 这一结果与文献中所示结果一致(网络 版附图1). 之后, 将DiART和iTRAQ标记样品中共同鉴 定的肽段的定量比值进行了统计. 如图3所示, DiART 和iTRAQ标记的肽段定量比值分布均以 2 为中心, 符合 理论比值4 (取 $\log _{2}$ 后为2), 说明两种标记方法均可得到 预期定量结果, 但相较于iTRAQ而言, DiART标记肽段 的定量比值分布更为集中, 其中 $76.16 \%$ 肽段比值为 1.8 2.2, 高于ITRAQ标记的肽段( $33.03 \%$ 肽段比值为 1.8 2.2), 且图3A中点的离散度明显低于图4B, 这说明 DiART方法定量稳定性和准确性优于iTRAQ.

之后根据鉴定到蛋白质的丰度情况对DiART和 iTRAQ标记定量方法的稳定性进行了进一步分析. DiART标记的E. coli样品中鉴定蛋白质(484个)的丰度 (empai值)范围为 $0.02 ~ 2.22$, iTRAQ标记的 $E$. coli样品 中鉴定蛋白质(571个)的丰度(empai值)范围为 $0.03 \sim 9.4$ (网络版附表1). 如果将empai $\leq 0.1$ 的蛋白定义为低丰度 蛋白, $0.1<\mathrm{empai} \leq 1.0$ 的定义为中等丰度蛋白, 而将empai $>1.0$ 的蛋白定义为高丰度蛋白, 则DiART标记样品 中鉴定的蛋白质分别有 $32.4 \%, 62.1 \%$ 和 $5.2 \%$ 位于低丰 度、中等丰度和高丰度区域，而iTRAQ标记样品中鉴 定的蛋白质分别有 $24.1 \%$ ，55.4\%和 $20.5 \%$ 位于低丰 度、中等丰度和高丰度区域。进一步分析发现， DiART标记样品中低、中、高丰度蛋白的定量比值中 位数分别为 $1.94,1.90$ 和 1.93 , 与理论值 2 的差值分别为 $0.06,0.10$ 和 0.07 ; 而iTRAQ标记样品中低、中、高丰 度蛋白的定量比值中位数分别为 $1.75,1.71$ 和 1.66 , 与 理论值 2 的差值分别为 $0.25,0.29$ 和 0.34 . 由以上数据可 见, 一方面, 虽然DiART标记的 E. coli样品中鉴定蛋白 质数目略低于iTRAQ标记, 但是有更高比例的蛋白质 


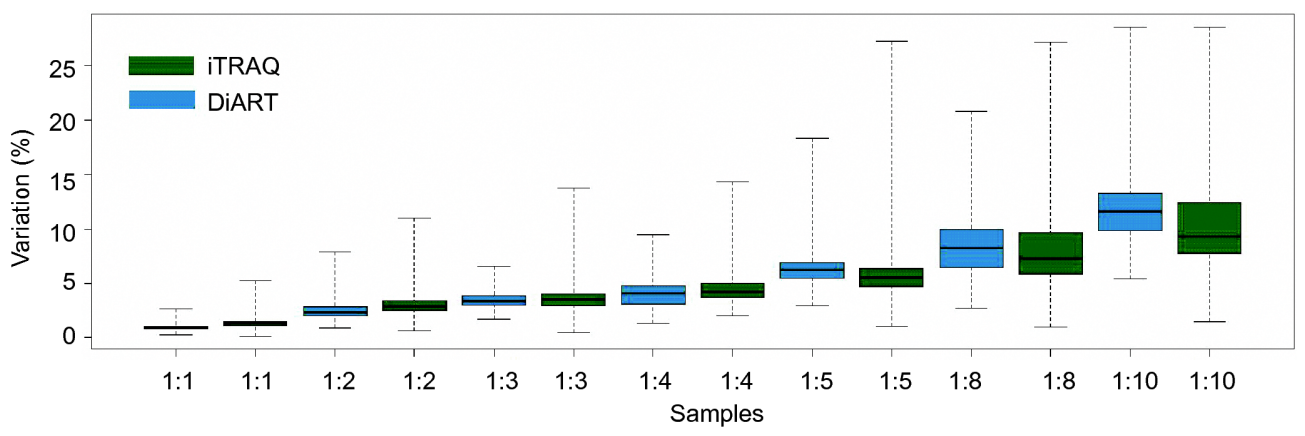

图 2 不同比例标记的BSA肽段的定量结果

蓝色标识的柱状代表DiART样品, 绿色标识的柱状代表iTRAQ样品
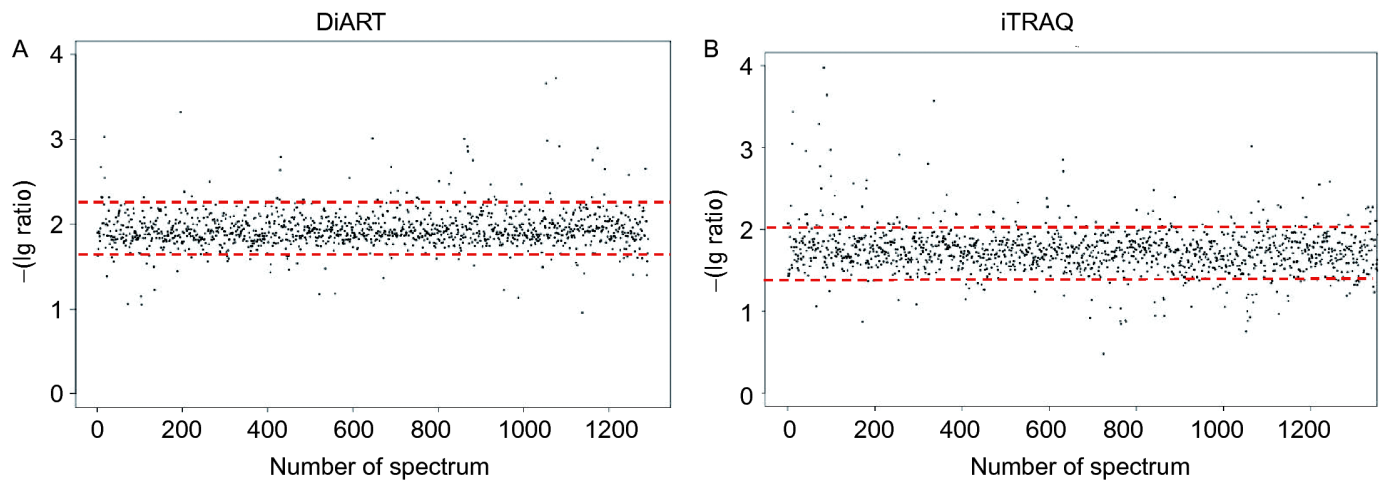

图 3 肽段定量比值分布

A: DiART标记肽段; B: iTRAQ标记肽段

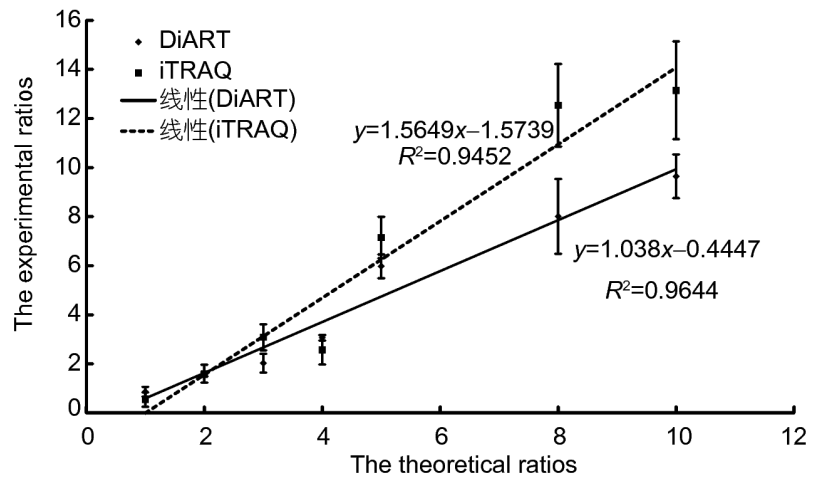

图 4 标记的BSA肽段在E. coli肽段背景中的定量结果

分布于中、低丰度区域，提示DiART标记方法对中、 低丰度蛋白的定量灵敏度高于iTRAQ方法; 另一方面， DiART标记样品中低、中丰度蛋白的定量比值中位数 较iTRAQ标记方法更接近于理论值2, 提示DiART标记 方法较iTRAQ方法定量稳定性更具优势.

\section{4 在复杂背景条件下DiART标记方法的抗干扰 能力}

接下来，为了分析DiART对复杂背景的抗干扰能 力, 将上述DiART和iTRAQ标记的BSA肽段各 7 个样品 分别混入到大肠杆菌样品中, 并进行质谱分析. 之后计 算出BSA各肽段的定量比值, 并将其与理论值比较. 如 图4所示，在大肠杆菌肽段背景中，DiART和iTRAQ标 记的BSA肽段定量结果与理论比值均呈现良好的线性 相关, 说明即使在复杂背景中, 两种方法均能在一定程 度上反映出目标蛋白的真实定量结果. 但从线性相关 程度来看, DiART标记的目标蛋白定量值与理论值的 线性相关系数为 0.96 , 高于iTRAQ标记的线性相关系 数 0.94 , 说明DiART定量测定值与真实值之间的线性 相关更好，结果更稳定. 另外，如果测定值与真实值相 同，那么斜率应该为 1 , 而DiART标记肽段定量结果的 斜率值为 1.03 , 与iTRAQ标记肽段的斜率值 1.56 相比前 
者更接近于理论斜率值 1 , 进一步说明DiART标记的目 标蛋白定量结果更接近真实值. 以上结果表明, DiART 标记方法更加耐受复杂背景的干扰.

\section{3 讨论}

一般来说, 在信号没有饱和的前提下, 报告离子的 强度越高, 肽段定量结果越可信. 基于这一理念, 就要 求标记试剂的报告基团比较容易从肽段上碎裂且能够 稳定存在形成报告离子. 之前的研究已经证实, DiART 标记试剂所标记的肽段在二级质谱中形成的报告离子

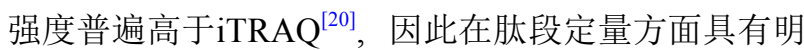
显优势. 然而, 从另一方面来讲, 报告离子强度得到保 证的同时可能会损失肽段其他碎片离子的强度，对肽 段鉴定造成一定影响. 因此，需要在低质量端报告离 子与肽段其他碎片离子之间达到一定的平衡，才能在 不影响鉴定的情况下得到高质量的定量结果. 在OrbiTrap类质谱仪中，通过改变碎裂能量可以调整肽段的 定性和定量结果，而在QTOF质谱仪中，对低质量端离 子的传输效率, 可显著影响肽段的定性和定量结果. 如 本研究所示, 当采用不同的碰撞池RF电压时, 肽段的 鉴定数目以及可定量肽段的数目出现了明显的变化, 其中DiART标记肽段鉴定数目最高达到 151 , 最低为 123 个, 而可定量肽段数目最高 145 个, 最低 118 个. 另
外, 不论是DiART还是iTRAQ, 在可定量肽段数目达到 最优的条件下，可鉴定肽段的数目都会有一定的减少， 因此在选取最优质谱方法时, 需要在二者之间进行平 衡，尽量选取不显著损失定性结果且定量结果达到最 优的方法.

本研究发现, DiART方法定量结果稳定性及动态 范围均高于iTRAQ方法, 其主要原因可能是由于 DiART报告基团更容易碎裂，因而得到的报告离子强 度普遍高于iTRAQ方法. 由于DiART标记肽段在进行 二级碎裂时会产生一个二级碳正离子中间体，其稳定 性高于iTRAQ标记肽段所产生的一级碳正离子中间 体. 而这些中间体在质谱仪环境中会重新排列最终形 成稳定的季胺报告离子. 这样最终体现的结果是 DiART有较高的报告离子强度. 也正是基于这一特点， 在iTRAQ类标记方法中普遍存在的定量结果低估问 题, 可被DiART在一定程度上克服 ${ }^{[21 ~ 23]}$. 在复杂样品背 景中, 由于DiART报告离子强度普遍较高, 因而受到其 他干扰离子的相对影响大大降低，达到对目标肽段更 为准确的定量结果.

综上所述，本研究通过对DiART标记的简单蛋白 样品、复杂蛋白样品以及复杂样品中目标蛋白的定量 分析，初步认为该标记方法的定量准确性、稳定性以 及受其他因素的干扰能力均有较好的表现，相对于 iTRAQ方法体现出了一定的优势.

致谢 感谢美国马里兰大学李舒伟老师提供了研究所需的DiART试剂. 本论文相关原始数据已上传蛋白质组综合资源 数据库 $\mathrm{P}$ ProX(http://www.iprox.org/), 项目编号为IPX0001088000.

\section{参考文献}

1 Välikangas T, Suomi T, Elo L L. A comprehensive evaluation of popular proteomics software workflows for label-free proteome quantification and imputation. Brief Bioinform, 2017, doi: 10.1093/bib/bbx054

2 Wen B, Zhou R, Feng Q, et al. IQuant: an automated pipeline for quantitative proteomics based upon isobaric tags. Proteomics, 2014, 14: 22802285

3 Domon B, Aebersold R. Options and considerations when selecting a quantitative proteomics strategy. Nat Biotechnol, 2010, 28: 710-721

4 Lindemann C, Thomanek N, Hundt F, et al. Strategies in relative and absolute quantitative mass spectrometry based proteomics. Biol Chem, 2017, 398: 687-699

5 Ong S E, Blagoev B, Kratchmarova I, et al. Stable isotope labeling by amino acids in cell culture, SILAC, as a simple and accurate approach to expression proteomics. Mol Cell Proteomics, 2002, 1: 376-386

6 Wiese S, Reidegeld K A, Meyer H E, et al. Protein labeling by iTRAQ: a new tool for quantitative mass spectrometry in proteome research. Proteomics, 2007, 7: 340-350 
7 Casey T M, Khan J M, Bringans S D, et al. Analysis of reproducibility of proteome coverage and quantitation using isobaric mass tags (iTRAQ and TMT). J Proteome Res, 2017, 16: 384-392

8 Frost D C, Greer T, Xiang F, et al. Development and characterization of novel 8-plex DiLeu isobaric labels for quantitative proteomics and peptidomics. Rapid Commun Mass Spectrom, 2015, 29: 1115-1124

9 Gygi S P, Rist B, Gerber S A, et al. Quantitative analysis of complex protein mixtures using isotope-coded affinity tags. Nat Biotechnol, 1999, 17: 994-999

10 Maccarrone G, Lebar M, Martins-de-Souza D. Brain quantitative proteomics combining GeLC-MS and isotope-coded protein labeling (ICPL). Methods Mol Biol, 2014, 1156: 175-185

11 Wang S, Kaltashov I A. A new strategy of using $\mathrm{O}^{18}$-labeled iodoacetic acid for mass spectrometry-based protein quantitation. J Am Soc Mass Spectrom, 2012, 23: 1293-1297

12 Krüger M, Moser M, Ussar S, et al. SILAC mouse for quantitative proteomics uncovers kindlin-3 as an essential factor for red blood cell function. Cell, 2008, 134: 353-364

13 Zanivan S, Krueger M, Mann M. In vivo quantitative proteomics: the SILAC mouse. Methods Mol Biol, 2012, 757: 435-450

14 Scholten A, Mohammed S, Low T Y, et al. In-depth quantitative cardiac proteomics combining electron transfer dissociation and the metalloendopeptidase Lys-N with the SILAC mouse. Mol Cell Proteomics, 2011, 10: O111.008474

15 Zeng D, Li S. Revival of deuterium-labeled reagents for protein quantitation. Chem Commun, 2009, 1: 3369

16 Wang J, Zhang Y, Xiang F, et al. Combining capillary electrophoresis matrix-assisted laser desorption/ionization mass spectrometry and stable isotopic labeling techniques for comparative crustacean peptidomics. J Chromatogr A, 2010, 1217: 4463-4470

17 Xiang F, Ye H, Chen R, et al. $N, N$-dimethyl leucines as novel isobaric tandem mass tags for quantitative proteomics and peptidomics. Anal Chem, 2010, 82: 2817-2825

18 Zhang R, Sioma C S, Thompson R A, et al. Controlling deuterium isotope effects in comparative proteomics. Anal Chem, 2002, 74: 3662-3669

19 Zhang J, Wang Y, Li S. Deuterium isobaric amine-reactive tags for quantitative proteomics. Anal Chem, 2010, 82: 7588-7595

20 Chen Z, Wang Q, Lin L, et al. Comparative evaluation of two isobaric labeling tags, DiART and iTRAQ. Anal Chem, 2012, 84: 2908-2915

21 Ow S Y, Salim M, Noirel J, et al. iTRAQ underestimation in simple and complex mixtures: "The good, the bad and the ugly". J Proteome Res, 2009, 8: 5347-5355

22 Savitski M M, Fischer F, Mathieson T, et al. Targeted data acquisition for improved reproducibility and robustness of proteomic mass spectrometry assays. J Am Soc Mass Spectrom, 2010, 21: 1668-1679

23 Karp N A, Huber W, Sadowski P G, et al. Addressing accuracy and precision issues in iTRAQ quantitation. Mol Cell Proteomics, 2010, 9: 18851897 


\title{
Evaluation of the use of DiART isobaric labeling tags in quantitative proteomics studies
}

\author{
JIANG CuiCui ${ }^{1}$, LV XiaoLei ${ }^{1}$, WU Di ${ }^{1}$, LI Yang ${ }^{1}$, DONG YuSheng ${ }^{1}$, ZHANG JiBin ${ }^{1} \&$ \\ WANG QuanHui ${ }^{1,2}$ \\ 1 Beijing Proteome Innovation, Beijing 101318, China; \\ 2 Sino-Danish College of University of Chinese Academy of Sciences, Beijing 100049, China
}

Isobaric labeling tags, such as iTRAQ (isobaric tags for relative and absolute quantification), are widely used in quantitative proteomics studies, in which peptides are chemically labeled and their abundance is represented by the intensity of reporter ions. DiART (deuterium isobaric amine reactive tag) is another type of reagent used for peptide labeling in quantitative proteomics studies, and although the reaction principal is similar to iTRAQ, it has a very different chemical structure. To evaluate the properties of DiART in peptide quantification, such as its accuracy, sensitivity, and dynamic range, we systematically analyzed different samples labeled with DiART using mass spectrometry. The quantitative analysis showed that the results of the peptides labeled with DiART were more accurate than those of the same peptides labeled with iTRAQ not only in the background of simple samples but also in that of complex samples, thus suggesting the usefulness of DiART in quantitative proteomics studies.

\section{DiART, iTRAQ, quantitative proteomics}

doi: $10.1360 / \mathrm{N} 052017-00152$ 